\title{
Comparison of sucrose metabolism in wheat seedlings during drought stress and subsequent recovery
}

\author{
F. NEMATI ${ }^{1}$, F. GHANATI ${ }^{1 *}$, H. AHMADI GAVLIGHI ${ }^{2}$, and M. SHARIFI ${ }^{1}$ \\ Department of Plant Biology, Faculty of Biological Science, Tarbiat Modares University, \\ 14115-154, Tehran, Iran ${ }^{1}$ \\ Department of Food Science and Technology, Faculty of Agriculture, Tarbiat Modares University, \\ 14115-336, Tehran, Iran $^{2}$
}

\begin{abstract}
Sucrose is a dominant sugar transported to the sink organs of a plant where it is metabolized to other compounds or stored. Here, the importance of sucrose metabolism in a drought-tolerant wheat cultivar was compared to a droughtsensitive one. The 4-d-old Triticum aestivum L. seedlings were exposed to drought induced by water cessation for $7 \mathrm{~d}$ and recovery after re-watering for further $7 \mathrm{~d}$. Under control conditions, constitutive expression of genes encoding vacuolar invertase $(V I)$ and sucrose synthase $(S u S)$ and activity of sucrose phosphate synthase (SPS) were significantly higher in the tolerant cultivar than in the sensitive one. Drought promoted the expressions of SPS and VI genes in the tolerant cultivar and increased their activities to 175 and $132 \%$, respectively, of those under control conditions. The activity of SuS and expression of its gene, however, were identical in both cultivars under drought stress. These changes resulted in more remarkable accumulation of sucrose in tolerant than in sensitive cultivar under water stress.
\end{abstract}

Additional key words: invertase, sucrose phosphate synthase, sucrose synthase, Triticum aestivum, water cessation.

Germinating seed and early seedlings are vulnerable to environmental stresses and most of the cereals are sensitive to water deficit in this stage. This vulnerability may be attributed, at least partly, to the fluctuations of soluble sugars during transition from heterotrophy to autotrophy. Soluble sugars do not only function as metabolic resources, but also act as signals regulating the plant response to water stress.

Wheat (Triticum aestivum L.) is the world most important cereal, which is widely grown in semi-arid areas, where drought as a severe abiotic stress occurs. It has been shown that the seedlings of spring wheat cultivars are able to tolerate severe drought up to fourth day after imbibition, but this capacity is not equal for all wheat genotypes (Bogdan and Zagdanska 2009, Koobaz et al. 2017).
In order to improve the survival under stress conditions, the drought tolerant plants comprise osmotic adjustments mainly mediated by nonstructural sugars (Rosa et al. 2009). Metabolism of sucrose is one of the key regulatory systems in drought tolerance (Bonfig et al. 2010). Sucrose stabilizes proteins and protects the cell during drought by the formation of an intracellular glass, which prevents cellular collapse (Koster 1991).

Sucrose synthesis in the cytosol is catalyzed by sucrose-phosphate synthase (SPS; EC 2.4.1.14) that synthesizes sucrose-6-phosphate from uridine diphosphate (UDP)-glucose and fructose 6-phosphate followed by dephosphorylation through sucrose-phosphate phosphatase (SPP; EC 3.1.3.24). The activity of SPS is often correlated with the rate of sucrose synthesis and sucrose is major transportable sugar (Fu et al. 2010).

Submitted 2 November 2017, last revision 24 January 2018, accepted 26 January 2018.

Abbreviations: NI - neutral invertase; SPP - sucrose-phosphate phosphatase; SPS - sucrose phosphate synthase; SuS - sucrose synthase; UDP - uridine diphosphate; VI - vacuolar invertase.

Acknowledgment: The authors would like to thank Tarbiat Modares University for providing the laboratory facilities and financial support for this project.

* Corresponding author; fax: (+98) 21 82884717, e-mail: ghangia@modares.ac.ir 
Cleavage of sucrose into hexoses is catalyzed by sucrose synthase (SuS; EC 2.4.1.13) and invertase (EC 3.2.1.26). Sucrose synthase (SuS), mainly found in the cytosol, reversibly synthesizes and degrades sucrose. Invertase irreversibly cleaves sucrose into glucose and fructose. Invertase activity is usually considered as a central modulator of assimilation partitioning and amplification of signals that control source-sink relationships in response to environmental changes (Hammond and White 2011). There are several invertase isozymes in higher plants. Acidic invertase is located in the vacuole (VI) or cell wall (Liao et al. 2017). Neutral/ alkaline invertase (NI) is present in the cytoplasm. Vacuolar invertase functions in the control of sugar composition and metabolic fluxes at the early stages of plant growth, and in sucrose partitioning at the later developmental stages (Roitsch and Gonzalez 2004). Abiotic stresses, e.g., water deficit, regulate the expression pattern of acidic invertase genes in different organs and developmental stages (Roitsch et al. 2003).

Effects of drought stress on sucrose metabolism in wheat during grain growth and development have been examined ( $\mathrm{Fu}$ et al. 2010). However, the putative mechanism(s) of sucrose status and efficacy in physiological events during germination, establishment, and seedlings growth are not well understood. In the present study, the influence of drought and recovery on sucrose content, enzymes of sucrose metabolism, and respective gene expressions were compared in 4-d-old seedlings of sensitive and tolerant wheat cultivars.

Two cultivars of spring wheat (Triticum aestivum L.), drought tolerant Sirvan and drought sensitive Marvdasht, were used (Saeedipour and Moradi 2011, PourAboughadareh et al. 2017). After routine surface sterilization, the seeds were allowed to germinate between two layers of filter paper moistened with distilled water in plastic transparent boxes under the dark for the first day and further $3 \mathrm{~d}$ under day/night temperatures of $25 / 20^{\circ} \mathrm{C}$, a relative humidity of $60 \%$, an 8-h photoperiod, and a photosynthetic photon flux density of $300 \mu \mathrm{mol} \mathrm{m} \mathrm{m}^{-2} \mathrm{~s}^{-1}$ at the plant level. Four-day-old seedlings of similar size were selected and subjected to drought stress by water cessation for $7 \mathrm{~d}$. Aliquots of samples were harvested and the others were re-watered with distilled water for recovery during further $7 \mathrm{~d}$. All samples were frozen in liquid nitrogen and stored at $-80^{\circ} \mathrm{C}$ for further analyses.

Extraction and determination of sugars were conducted following the method described by Verspreet et al. (2012, 2013). Sucrose, glucose, and fructose were determined by a high performance anion exchange chromatography with pulsed amperometric detection (HPAEC-PAD) system consisted of AZURA P $6.1 \mathrm{~L}$ pump (Knauer, Berlin, Germany), a DECADE Elite electrochemical detector (Antec, Leyden, The Netherlands) and Clarity Chrom 6.1.0 software for data acquisition. Separations were performed using a
CarboPacTM PA1 $(4 \times 250 \mathrm{~mm})$ analytical column (Dionex, Sunnyvale, CA, USA). Sugars were eluted isocratically by a $\mathrm{CO}_{2}$-free aqueous solution of $50 \mathrm{mM}$ $\mathrm{NaOH}$ at $32{ }^{\circ} \mathrm{C}$, and a flow rate was $1 \mathrm{~cm}^{3} \mathrm{~min}^{-1}$. The following pulse potentials and durations were used for detections: $\mathrm{E}_{1}=0.05 \mathrm{~V}, \mathrm{t}_{1}=500 \mathrm{~ms} ; \mathrm{E}_{2}=0.75 \mathrm{~V}$, $\mathrm{t}_{2}=130 \mathrm{~ms} ; \mathrm{E}_{3}=0.8 \mathrm{~V}, \mathrm{t}_{3}=120 \mathrm{~ms}$, data-collection rate: $1.33 \mathrm{~Hz}$. All enzyme extraction operations were performed at $4{ }^{\circ} \mathrm{C}$. The activity of VI and NI were measured according to Merlo and Passera (1991). The activities of SPS and SuS were evaluated by measuring absorbance of sucrose at $520 \mathrm{~nm}$ by a spectrophotometer (Cintra 6, GBC, Melbourne, Australia) according to the method of Huber et al. (1984).

Semi-quantitative reverse transcription (RT)-PCR technique was used for detection of relative expressions of SPS3, SuS1 and VI using $18 S$ rRNA as an internal control. Total RNA from frozen samples was extracted by TRIzol® reagent (Ambion Waltham, MA, USA) following the manufacturer's instructions. The purified RNA $(3 \mu \mathrm{g})$ was used for synthesis of the first strand cDNA using oligo dT18 as a primer (MWG-Biotech $A G$, Ebersberg, Germany) and the first-strand cDNA synthesis kit (Fermentas, Lithuania) according to the manufacturer's instructions. PCR was performed in $20 \mathrm{~mm}^{3}$ of reaction mixture containing $0.3 \mathrm{~mm}^{3}$ of first-strand cDNA, $0.5 \mathrm{U}$ of recombinant TaqDNA polymerase (CinnaGen, Tehran, Iran), $10 \mathrm{mM}$ Tris- $\mathrm{HCl}$ (pH 8.8), 50 $\mathrm{mM} \mathrm{KCl}, \quad 1.5 \mathrm{mM} \quad \mathrm{MgCl}_{2}, 200 \mu \mathrm{M}$ of each deoxynucleoside triphosphate, and $2 \mu \mathrm{M}$ of forward and reverse primers (Table 1 Suppl.). Forward and reverse specific primers of Haematococcus pluvialis strain TMU1 were used to amplify the coding region of $18 \mathrm{~S} r R N A$ (NCBI accession No. KT601294) gene. The primers of SPS3, SuS1, and VI were designed according to the sequences cited in NCBI under accession numbers of AF347065.1, AJ001117.1, and AF069309, respectively.

Specific primers for the genes involved in sucrose metabolism were designed using PrimerQuest from IDT SciTools (Table 1 Suppl.). Products of PCR were separated by $1 \%(\mathrm{~m} / \mathrm{v})$ agarose gel electrophoresis. The band intensity on the gel stained with ethidium bromide was measured by UV documentation luminescent image analysis (UVITEC, Cambridge, England) and then quantified by Image $J$ v. 1.4.3.67 software (National Institutes of Health, Bethesda, MD, USA,). The nucleotide sequences of amplified fragments were confirmed by DNA bidirectional sequencing using specific primers (Macrogen, Seoul, Korea). Band intensity was expressed as relative absorbance units. Normalization with respect to a positive control $18 S$ rRNA was calculated to normalize variations in sample concentration and as a control for reaction efficiency.

All of the experiments and observations were performed in a completely randomized design with three independent repetitions using at least nine samples each. 
Statistical analyses were performed using analysis of variance $(A N O V A)$ by SPSS v. 16 software (Chicago, IL, USA). The significance of differences between treatments was evaluated using the least significant difference (LSD) test at $P \leq 0.05$.

Table 1. Content of sugars $\left[\mu \mathrm{mol} \mathrm{g}^{-1}\right.$ (d.m.)], activities of sucrose phosphate synthase (SPS) [mmol(sucrose) $\mathrm{g}^{-1}\left(\mathrm{~d} . \mathrm{m}\right.$.) $\mathrm{min}^{-1}$, sucrose synthase $(\mathrm{SuS}),\left[\mathrm{mmol}(\right.$ sucrose $) \mathrm{g}^{-1}$ (d.m.) $\mathrm{min}^{-1}$, neutral invertase (NI) [mmol(glucose) $\mathrm{g}^{-1}$ (d.m.) $\mathrm{min}^{-1}$ ], and vacuolar invertase (VI) $\left[\mathrm{mmol}\right.$ (glucose) $\mathrm{g}^{-1}$ (d.m.) $\left.\mathrm{min}^{-1}\right]$, and relative expressions of SPS3, SuS1, and VI genes (using $18 S$ rRNA as an internal control) in sensitive (Marvdasht) and tolerant (Sirvan) wheat cultivars before treatment $(\mathrm{Ctrl})$, after $7 \mathrm{~d}$ of drought stress (DS), and after $7 \mathrm{~d}$ of recovery after re-watering (RW). Means \pm SDs, $n=3$. Different letters show significant differences at $P \leq 0.05$ according to LSD test.

\begin{tabular}{|c|c|c|c|}
\hline \multirow{2}{*}{$\begin{array}{l}\text { Parameter } \\
\text { Glucose }\end{array}$} & \multirow{2}{*}{$\begin{array}{l}\text { Treatment } \\
\text { Ctrl }\end{array}$} & \multicolumn{2}{|c|}{ Drought sensitiveDrought tolerant } \\
\hline & & $4.08 \pm 0.83^{\mathrm{d}}$ & $34.51 \pm 0.21^{\mathrm{a}}$ \\
\hline & DS & $21.08 \pm 1.63^{\mathrm{b}}$ & $13.11 \pm 0.77^{\mathrm{c}}$ \\
\hline & RW & $13.48 \pm 0.82^{\mathrm{c}}$ & $19.46 \pm 1.64^{b}$ \\
\hline \multirow[t]{3}{*}{ Fructose } & Ctrl & $112.04 \pm 0.87^{\mathrm{a}}$ & $9.40 \pm 3.77^{\mathrm{c}}$ \\
\hline & DS & $33.56 \pm 0.31^{\mathrm{b}}$ & $4.25 \pm 1.41^{\mathrm{d}}$ \\
\hline & RW & $5.13 \pm 0.34^{\mathrm{d}}$ & $4.74 \pm 0.30^{\mathrm{d}}$ \\
\hline \multirow[t]{3}{*}{ Sucrose } & Ctrl & $0.81 \pm 0.00^{\mathrm{e}}$ & $1.55 \pm 0.02^{\mathrm{e}}$ \\
\hline & DS & $16.60 \pm 0.34^{\mathrm{b}}$ & $42.97 \pm 0.01^{\mathrm{a}}$ \\
\hline & RW & $5.36 \pm 0.92^{\mathrm{d}}$ & $9.96 \pm 0.80^{\mathrm{c}}$ \\
\hline \multirow[t]{3}{*}{ SPS } & Ctrl & $0.02 \pm 0.001^{\mathrm{c}}$ & $0.03 \pm 0.002^{\mathrm{b}}$ \\
\hline & DS & $0.02 \pm 0.004^{\mathrm{c}}$ & $0.05 \pm 0.005^{\mathrm{a}}$ \\
\hline & RW & $0.02 \pm 0.002^{\mathrm{c}}$ & $0.02 \pm 0.004^{\mathrm{c}}$ \\
\hline \multirow[t]{3}{*}{$\mathrm{SuS}$} & Ctrl & $0.28 \pm 0.04^{\mathrm{b}}$ & $0.31 \pm 0.04^{\mathrm{b}}$ \\
\hline & DS & $1.96 \pm 0.08^{\mathrm{a}}$ & $1.60 \pm 0.05^{\mathrm{a}}$ \\
\hline & RW & $0.40 \pm 0.02^{\mathrm{b}}$ & $0.34 \pm 0.03^{\mathrm{b}}$ \\
\hline \multirow[t]{3}{*}{ NI } & Ctrl & $6.48 \pm 0.08^{\mathrm{b}}$ & $4.95 \pm 0.47^{\mathrm{d}}$ \\
\hline & DS & $8.89 \pm 0.57^{\mathrm{a}}$ & $6.33 \pm 0.91^{\mathrm{bc}}$ \\
\hline & RW & $5.11 \pm 0.11^{\mathrm{cd}}$ & $5.73 \pm 0.55^{\mathrm{bc}}$ \\
\hline \multirow[t]{3}{*}{ VI } & Ctrl & $21.87 \pm 1.26^{\mathrm{a}}$ & $14.31 \pm 1.15^{\mathrm{b}}$ \\
\hline & DS & $14.30 \pm 1.72^{b}$ & $21.73 \pm 4.10^{\mathrm{a}}$ \\
\hline & RW & $12.53 \pm 1.12^{\mathrm{b}}$ & $15.75 \pm 1.20^{\mathrm{b}}$ \\
\hline \multirow[t]{3}{*}{ SPS3/18S rRNA } & Ctrl & $0.41 \pm 0.02^{\mathrm{c}}$ & $0.88 \pm 0.08^{\mathrm{b}}$ \\
\hline & DS & $0.88 \pm 0.06^{\mathrm{b}}$ & $1.14 \pm 0.02^{\mathrm{a}}$ \\
\hline & RW & $0.45 \pm 0.02^{\mathrm{c}}$ & $0.34 \pm 0.03^{c}$ \\
\hline \multirow[t]{3}{*}{ SuS1/18S rRNA } & Ctrl & $0.15 \pm 0.01^{\mathrm{c}}$ & $0.46 \pm 0.02^{\mathrm{a}}$ \\
\hline & DS & $0.43 \pm 0.03^{\mathrm{ab}}$ & $0.38 \pm 0.02^{\mathrm{b}}$ \\
\hline & RW & $0.48 \pm 0.05^{\mathrm{a}}$ & $0.39 \pm 0.01^{\mathrm{b}}$ \\
\hline \multirow[t]{3}{*}{ VI/18S rRNA } & Ctrl & $0.69 \pm 0.09^{c}$ & $1.56 \pm 0.01^{\mathrm{b}}$ \\
\hline & DS & $0.57 \pm 0.18^{\mathrm{c}}$ & $1.88 \pm 0.19^{\mathrm{a}}$ \\
\hline & RW & $1.34 \pm 0.08^{b}$ & $1.53 \pm 0.04^{b}$ \\
\hline
\end{tabular}

In the sensitive wheat cultivar, glucose content significantly increased under water stress but it was reduced after re-watering, although it was higher than under control conditions (Table 1). A similar pattern was observed in sucrose content, whereas fructose showed reducing tendency during water stress and after rewatering (Table 1). In the tolerant cultivar, the content of glucose and fructose significantly decreased under drought stress and did not restore to the control level after re-watering (Table 1). Sucrose content of this cultivar remarkably increased during water stress and decreased after re-watering (Table 1).

No change was observed in the activity of SPS in the sensitive cultivar but in the tolerant cultivar, SPS activity significantly increased under drought stress and decreased after re-watering (Table 1). In well-watered samples, the activity of $\mathrm{SuS}$ was identical in both cultivars and it increased under drought stress, but declined to the control level after re-watering (Table 1). Under control conditions, activities of both NI and VI were higher in the sensitive cultivar than in the tolerant one (Table 1). The activity of NI in both cultivars increased after drought stress but only in the sensitive cultivar decreased after re-watering (Table 1). The activity of VI in the tolerant wheat cultivar increased under water stress but after re-watering returned to the control level (Table 1). Whereas in the sensitive cultivar, VI activity decreased under drought stress and rewatering, compared to the control conditions (Table 1).

Semi-quantitative RT-PCR analysis showed that under control conditions, expressions of SPS3, SuS1, and $V I$ genes in the tolerant cultivar were higher than in the sensitive one (Table 1). Expression of SPS3 gene in both cultivars increased under drought stress but returned to the level of control conditions after re-watering (Table 1). Expression of SuS1 in the sensitive cultivar increased under drought and remained constant after re-watering. Expression of this gene in the tolerant cultivar, however, decreased under water stress and remained decreased even after re-watering (Table 1). Expression of $V I$ in the tolerant cultivar remarkably increased after water cessation and returned to the control level after rewatering (Table 1). In the sensitive cultivar, however, no significant change was observed in VI activity under water stress but it increased after re-watering (Table 1).

Germination is a unique developmental transition from a metabolically quiescent seed to an actively growing seedling. Early seedling development has a limited carbon supply because of photosynthetic system which is not yet active (Yu et al. 2015).

During intensive growth phase of seedlings, glucose may act as a signal for increased respiration, which provides the cells with carbon and energy (Miazek et al. 2001). Therefore, declined content of soluble glucose and fructose of the tolerant cultivar under water stress can be interpreted as a more sustainable metabolism. At the same conditions, increased content of glucose of the sensitive cultivar denoted reduced glucose consumption and limited growth.

Under drought stress, modification of carbon partitioning in favour of sucrose synthesis resulted in accumulation of sucrose in both cultivars as a mechanism of acclimation to water deficiency. Fluctuation of sucrose content was coincident with the changes in activities of 
key enzymes controlling sucrose metabolism (SPS, SuS, VI, and NI) and expressions of their genes.

Previous studies have shown an overlap in the expression patterns of the different SPS gene families in wheat, among which SPS III (family D) genes show the highest expression in germinating seedlings (Castleden et al. 2004). In the present study, in the sensitive cultivar, expression of the SPS gene increased under water stress but SPS activity did not change and sucrose content increased. This suggested that this sucrose was originated either from its continuous translocation from the grain or resulted due to the $\mathrm{SuS}$ activity. The $\mathrm{SuS}$ is a bifunctional enzyme, whose catalytic activity provides the cell with UDP-glucose moieties, mainly for cellulose synthesis, and also has anabolic activity resulting in sucrose production. Regard to the procedure followed in this paper, synthetic activity of SuS was measured. So far, two biochemically similar isozymes, SuS1 and SuS2, have been recognized. The expression of $S u S 1$ has shown predominance in wheat roots and leaves in response to abiotic stresses (Marafla et al. 1990). As expected, water cessation increased the SuS1 gene expression and its activity in the sensitive cultivar. The results indicated that under energy-limiting conditions, this wheat cultivar changed its energy expenditure toward the biosynthesis of sucrose and less to cell growth and biosynthesis of cellulose.

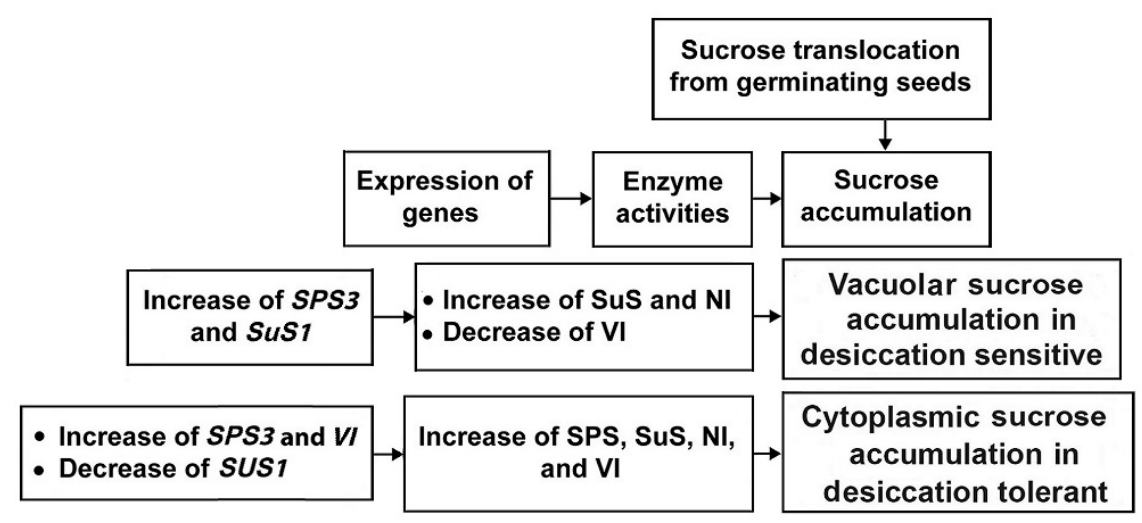

Fig. 1. A schematic model summarizing the role of sucrose metabolism in the tolerance or sensitivity of 4-d-old wheat seedlings to water stress and recovery.

Utilization of sucrose as a carbon source and its accumulation is controlled by the activity of invertases. Plant invertases occupy a pivotal position in the balance between the different sugar signals generated by imported sucrose. The fate of the vacuolar sucrose is essentially controlled by the differential activities of VI and NI (Devi et al. 1984). The VI regulates osmotic pressure, sugar signals, and sucrose accumulation, especially during the expansion phases of sink organs (Koch 2004). In the sensitive wheat cultivar tested in the present study, the expression of VI and VI activity decreased under water stress, but the activity of NI and sucrose content increased. It may be related to the fact that in comparison with VI, cytoplasmic invertases are minimally active in most systems, and cytoplasmic sucrose is frequently transported into vacuoles for cleavage (Winter and Huber 2000).

In the tolerant cultivar during water stress, an increase of SPS3 gene expression and its activity was accompanied by an accumulation of sucrose. The expression of $\mathrm{SuSl}$ gene in the tolerant cultivar decreased under water stress. It seems, however, that this expression was enough to keep the activity high. In spite of an increase of invertases activities during water depletion in the tolerant wheat cultivar, sucrose content was high. Medici et al. (2014) also reported such incongruity between VI activity and sucrose content. Although transport of sucrose from cytoplasm to vacuole was out of the scope of the present research, the results suggested that in the tolerant cultivar, sucrose was predominantly synthesized in cytoplasm and kept there, out of access of VI but available for NI, whose contribution to hydrolysis of sucrose is very low (Koch 2004).

Our present results are summarized in Fig. 1. They demonstrated that drought tolerant seedlings favour sucrose synthesis in order to enhance energy saving and adjust cellular metabolism at both metabolic and gene expression levels. In addition, rapid metabolism of sucrose as an immediate energy source during rewatering suggested that its accumulation could be used as a biomarker in the selection for improved drought tolerance and post-drought recovery in wheat species.

In conclusion, it is likely that accumulation of sucrose is a unique tolerance characteristic of wheat cultivars. The same characteristic is usually observed in resurrection plants during drought (Djilianov et al. 2011). High content of sucrose in wheat cultivars, however, follows two distinct routes with two distinct results. In the tolerant cultivar, cytoplasmic sucrose synthesis and accumulation is much higher than in the sensitive cultivar, whereas in the sensitive cultivar, vacuolar sucrose content is high due to low VI cleavage activity. 


\section{References}

Bogdan, J., Zagdanska, B.: Alterations in sugar metabolism coincide with a transition of wheat seedlings to dehydration intolerance. - Environ. exp. Bot. 66: 186-194, 2009.

Bonfig, K.B., Gabler, A., Simon, U.K., Luschin-Ebengreuth, N., Hatz, M., Berger, S., Muhamma, N., Zeier, J., Sinha, A.K., Roitsch, T.: Post-translational derepression of invertase activity in source leaves via down-regulation of invertase inhibitor expression is part of the plant defense response. Mol. Plant 3: 1037-1048, 2010.

Castleden, C.K., Aoki, N., Gillespie, V.J., Mac Rae, E.A., Quick, W.P., Buchner, P., Foyer, C.H., Furbank, R.T., Lunn, J.E.: Evolution and function of the sucrose-phosphate synthase gene families in wheat and other grasses. - Plant Physiol. 135: 1753-1764, 2004.

Devi, M., Tenaud, P., Champigny, M.L.: A comparative study on the soluble invertase activities and sucrose content in different clones of sugar cane. - In: Sybesma, C. (ed.): Advances in Photosynthesis Research. Advances in Agricultural Biotechnology. Vol. 4. Pp. 881-884. Springer, Dordrecht 1984.

Djilianov, D., Ivanov, S., Moyankova, D., Miteva, L., Kirova, E., Alexieva, V., Joudi, M., Peshev, D., Van den Ende, W.: Sugar ratios, glutathione redox status and phenols in the resurrection species Haberlea rhodopensis and the closely related non-resurrection species Chirita eberhardtii. - Plant Biol. 13: 767-776, 2011.

Fu, J., Huang, B., Fry, J.: Osmotic potential, sucrose level, and activity of sucrose metabolic enzymes in tall fescue in response to deficit irrigation. - J. amer. Soc. hort. Sci. 135: 506-510, 2010.

Hammond, J.P., White, P.J.: Sugar signaling in root response to low phosphorus availability. - Plant Physiol. 156:10331040, 2011.

Huber, S.C., Rufty, T.W., Kerr, P.S.: Effect of photoperiod on photosynthate partitioning and diurnal rhythms in sucrose phosphate synthase activity in leaves of soybean (Glycine max L. [Merr.]) and tobacco (Nicotiana tabacum L.). - Plant Physiol. 75: 1080-1084, 1984.

Koch, K.: Sucrose metabolism: regulatory mechanisms and pivotal roles in sugar sensing and plant development. - Curr. Opin. Plant Biol. 7: 235-246, 2004.

Koobaz, P., Ghanati, F., Hosseini Salekdeh, G., Moradi, F., Hadavand, H.: Drought tolerance in four-day-old seedlings of a drought-sensitive cultivar of wheat. - J. Plant Nutr. 40: 574-583, 2017.

Koster, K.L.: Glass formation and desiccation tolerance in seeds. - Plant Physiol. 96: 302-304, 1991.

Liao, W.B., Li, Y.Y., Lu, C., Peng, M.: Expression of sucrose metabolism and transport genes in cassava petiole abscission zones in response to water stress. - Biol. Plant. 61: 219-226, 2017.
Marafla, C., Garcia-Olmedo, F., Carbenero, P.: Differential expression of two types of sucrose synthase-encoding genes in wheat in response to anaerobiosis, cold shock and light. Gene 88: 167-172, 1990.

Medici, A., Laloi, M., Atanassova, R.: Profiling of sugar transporter genes in grapevine coping with water deficit. FEBS Lett. 588: 3989-3997, 2014.

Merlo, L., Passera, C.: Changes in carbohydrate and enzyme levels during development of leaves of Prunus persica, a sorbitol synthesizing species. - Physiol. Plant. 83: 621-626, 1991.

Miazek, A., Bogdan, J., Zagdanska, B.: Effects of water deficit during germination of wheat seeds. - Biol. Plant. 44: 397403, 2001.

Pour-Aboughadareh, A., Ahmadi, J., Mehrabi, A.A., Etminan, A., Moghaddam, M., Siddique, K.H.: Physiological responses to drought stress in wild relatives of wheat: implications for wheat improvement. - Acta Physiol. Plant. 39: 1-16, 2017.

Roitsch, T., Balibrea, M.E., Hofmann, M., Proels, R., Sinha, A.K.: Extracellular invertases: key metabolic enzyme and PR protein. - J. exp. Bot. 54: 513-24, 2003.

Roitsch, T., Gonzalez, M.C.: Function and regulation of plant invertases: sweet sensations. - Trends Plant Sci. 9: 606-613, 2004.

Rosa, M., Prado, C., Podazza, G., Interdonato, R., Gonzalez, J.A., Hilal, M. Prado F.E.: Soluble sugars, metabolism, sensing and abiotic stress. - Plant Signal. Behav. 4: 388-393, 2009.

Saeedipour, S., Moradi, F.: Comparison of the drought stress responses of tolerant and sensitive wheat cultivars during grain filling: impact of invertase activity on carbon metabolism during kernel development. - J. agr. Sci. 3: 3244, 2011.

Verspreet, J., Cimini, S., Vergauwen, R., Dornez, E., Locato, V., Le Roy, K., De Gara, L., Van den Ende, W., Delcour, J.A., Courtin, C.M.: Fructan metabolism in developing wheat (Triticum aestivum L.) kernels. - Plant Cell Physiol. 54: 2047-2057, 2013.

Verspreet, J., Pollet, A., Cuyvers, S., Vergauwen, R., Van den Ende, W., Delcour, J.A., Courtin, C.M.: A simple and accurate method for determining wheat grain fructan content and average degree of polymerization. - J. agr. Food Chem. 60: 2102-2107, 2012.

Winter, H., Huber, S.C.: Regulation of sucrose metabolism in higher plants: localization and regulation of activity of key enzymes. - Crit. Rev. Biochem. mol. Biol. 35: 253-289, 2000.

Yu, S.M., Lo, S.F., Ho, T.H.D.: Source-sink communication: regulated by hormone, nutrient, and stress cross signaling. Trends Plant Sci. 20: 844-857, 2015 